\title{
A novel polymer-surfactant complex mixture to improve diesel fuel flow in a rotating disk apparatus
}

\begin{abstract}
This paper introduces a novel complex system to decrease the polymer degradation using polyisobutylene (PIB) and sodium lauryl ether sulfate (SLES). These materials are tested individually and as a complex mixture in a rotating disk apparatus (RDA) at various concentrations and rotational speeds (rpm). From the experimental results, it can be observed that the drag reduction for the complex mixture of cationic polymer with anionic surfactant has a better performance than the reduction of individual polymer or surfactant, respectively. This can be as a result of the important role played by complex mixtures that are highly dependent on alkyl chain in the surfactant. The maximum \%DR observed in laminar flow was $38.42 \%$ for complex mixture at $1000 \mathrm{rpm}$, while the $\% \mathrm{DR}$ of polyisobutylene and sodium lauryl ether sulfate at the same condition were $27.36 \%$ and $28.42 \%$, respectively.
\end{abstract}

Keyword: Polymer; Surfactants; Drag reduction; Complex mixtures; Rotating disk apparatus (RDA) 